\title{
Resolution of a large periapical lesion in an immature maxillary lateral incisor with the aid of triple antibiotic paste
}

SADJ October 2021, Vol. 76 No. 9 p560 - p564

N Potgieter, ${ }^{1}$ GD Buchanan ${ }^{2}$

\begin{abstract}
Introduction

Apexification procedures are frequently performed on immature permanent teeth with incomplete root formation, open apices and necrotic pulp status with or without periapical lesions in order to induce a calcific barrier prior to root canal therapy. The elimination and control of infection in the root canal space is critical to the success of these procedures.
\end{abstract}

\section{Case Description}

A healthy 21-year old male presented with pulpal necrosis, a large periapical lesion, incomplete root formation and an open apex on a maxillary right lateral incisor. Triple antibiotic paste was used to achieve antimicrobial control after traditional calcium hydroxide paste medicament failed to resolve the symptoms. Obturation was achieved using MTA and the conventional apexification technique. Excellent healing of the large periapical lesion was achieved without surgical intervention and the 4-year follow-up CBCT demonstrated complete bone fill of the lesion.

\section{Conclusion}

Clinicians should be aware that alternative antimicrobial medicaments, such as triple antibiotic paste, may be beneficial in situations where conventional medicaments prove unsuccessful. The use of triple antibiotic paste may result in sufficient healing of the periapical lesion to justify placement of an MTA apical barrier without the need for surgical intervention.

Author affiliations:

1. Nicoline Potgieter: BChD, PDD, PGDip (Dent), MSc (Dent) Department of Paediatric Dentistry, Faculty of Dentistry, University of the Western Cape. ORCID:0000-0003-4061-3322

2. Glynn Buchanan: BChD, PDD, MSc (Dent) Department of Odontology, School of Dentistry, Faculty of Health Sciences, University of Pretoria. ORCID:0000-0003-2957-166X

Corresponding author: Nicoline Potgieter

Department of Paediatric Dentistry. University of the Western Cape,

Faculty of Dentistry, Francie Van Zijl Drive, Parow, 7505, South Africa

E-mail: nipotgieter@uwc.ac.za

Telephone: +27219373107

The role played and the respective contribution:
1. Nicoline Potgieter: $50 \%$

2. Glynn Buchanan: $50 \%$
Keywords

apexification, endodontics, triple antibiotic paste, calcium hydroxide.

\section{INTRODUCTION}

Apexification is a term referring to endodontic procedures performed to achieve apical closure of immature permanent teeth presenting with incomplete root development, open apices and pulpal necrosis. ${ }^{1}$

Historically, apexification involved the placement of calcium hydroxide $(\mathrm{CH})$ paste over multiple treatment appointments in order to create a calcific barrier in the apical area prior to the completion of root canal treatment. ${ }^{2}$ Whilst this approach demonstrated clinical success, several disadvantages were reported with the technique, including: difficulty with patient follow-up, delayed treatment, risk of root fracture and unpredictability of the apical seal. ${ }^{1}$ Additionally, placement of $\mathrm{CH}$ for extended periods has been demonstrated to weaken tooth structure. ${ }^{3}$

In modern endodontics, calcium silicate cements such as mineral trioxide aggregate (MTA) are recommended as the preferred material for use in apexification procedures due to their excellent sealing properties, biocompatibility ${ }^{4}$ and antimicrobial activity. ${ }^{5}$ The use of calcium silicate cements has additionally led to the ability to complete apexification procedures in a single visit. ${ }^{6}$

Whilst single-visit apexification approaches may be beneficial to both patients and practitioners in terms of decreased time and a reduced need for follow-up/patient compliance, great care must be taken to ensure that adequate disinfection of the root canal space is achieved prior to obturation. Triple antibiotic paste (TAP), first described by Sato et al. ${ }^{7}$, is an effective antimicrobial agent, consisting of a mixture of ciprofloxacin, metronidazole and minocycline and has been successfully used in regenerative endodontic procedures for permanent teeth demonstrating pulpal necrosis. ${ }^{8,9}$

The following case reports the successful endodontic management of a maxillary lateral incisor with a large periapical lesion and open apex, where TAP was used for root canal disinfection after traditional $\mathrm{CH}$ paste failed to achieve adequate microbial control prior to obturation. 


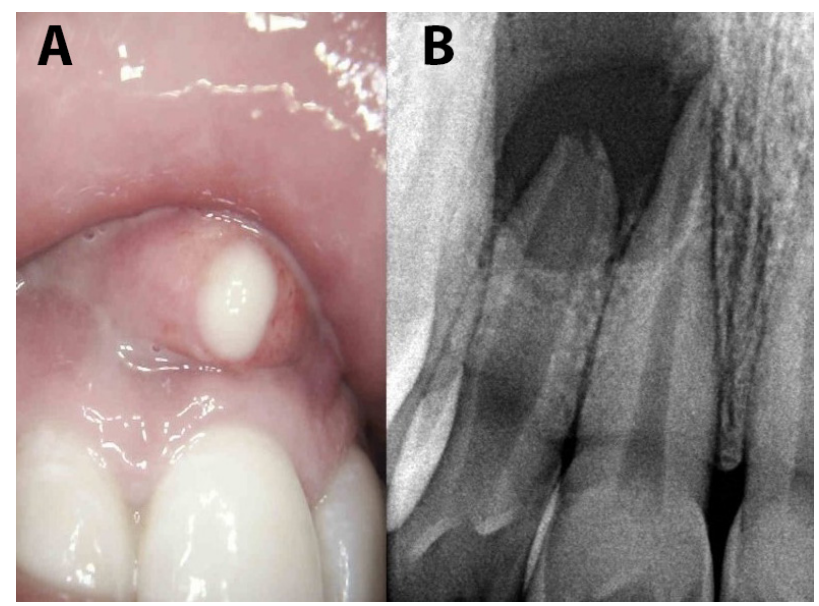

Figure 1. Pre-operative evaluation of the right maxillary lateral incisor: A) clinical image of suppurative swelling and B) peri-apical radiograph

Ethical approval for this case report was obtained from the Research Ethics Committee, Faculty of Health Sciences, University of Pretoria (Protocol number: 19/2021).

\section{Case presentation}

A healthy, 21-year old male presented to the University of Pretoria Oral Health Centre with acute suppurative swelling and pain in the right pre-maxillary area (Figure 1a). Radiographic examination confirmed an open apex and large periapical radiolucency associated with right maxillary lateral incisor (Figure 1b).

The tooth was anesthetized, isolated with a rubberdam and an emergency root canal treatment was performed for pain relief. Pus drainage was established through the canal and buccal soft tissue during copious irrigation with a saline solution. Digital pressure was applied to the buccal swelling to further express inflammatory exudates. Chlorhexidine ( $2 \%$ concentration) was chosen as the preferred irrigant, considering the absence of an apical stop and increased risk of sodium hypochlorite extrusion and injury. Calcium hydroxide paste (Calacept, Ultradent, South Jordan, USA) was placed as an intracanal medicament and the access cavity temporarily sealed with glass-ionomer cement, placed on clean dentine and enamel. (Fuji IX GP, GC Corporation, Tokyo, Japan).

The patient was referred for a small-volume cone-beam computed tomography (CBCT) scan. The scan revealed a periapical lesion with well-defined borders, ballooning appearance, radiolucent lumen, perforation of the cortical plate, size greater than $10 \mathrm{~mm}$ in diameter and open apex of the right maxillary lateral incisor (Figure 2). Differential diagnosis included either a large periapical granuloma or radicular cyst. The initial treatment plan included apexification, obturation of the canal and - if required at a later stage cystectomy or periapical surgery. The final diagnosis was to be confirmed by biopsy and histopathological evaluation.

At the subsequent follow-up visit, no swelling or drainage was clinically visible however the patient reported continued symptoms of discomfort. Following anaesthesia and rubber dam isolation, the canal was cleared of $\mathrm{CH}$ medicament and working length was determined by an apex locator (ProPex Pixi, Dentsply Sirona, Ballaigues, Switzerland) and confirmed with a radiograph. Negative pressure irrigation with 6\% sodium hypochlorite (Chlor-Xtra, Vista Dental, Racine, USA) was performed using the Endovac irrigation system (Kerr Corp., California, USA) during instrumentation of the canal with a size \#90 k-file. After instrumentation, $17 \%$ ethylenediaminetetraacetic acid (EDTA, Vista Dental, Racine, USA) irrigation was used followed by a final rinse of $\mathrm{NaOCl}$. When attempting to dry the canal with paper points, persistent clear fluid drainage was found.

A triple antibiotic paste (TAP), consisting of a mixture of metronidazole, ciprofloxacin and minocycline was mixed in a 1:1:1 ratio with propylene glycol as carrier and placed into the canal using a k-file and anticlockwise rotation. Precautions were taken to place the TAP beneath the level of the cemento-enamel junction to prevent subsequent tooth discoloration of the crown. The access cavity was temporarily sealed with glass-ionomer cement (Fuji IX GP, GC Corporation, Tokyo, Japan) placed on clean dentine and enamel.

The patient failed to return for follow-up visits in the subsequent months due to personal circumstances. Numerous attempts were made to contact the patient, motivating a return for follow-up and completion of the treatment. The patient finally returned after 2 years and 3 months. At this visit, the patient was asymptomatic and radiographic evaluation demonstrated sufficient healing of the periapical lesion around the maxillary right lateral incisor to justify placement of a Mineral Trioxide Aggregate (MTA) apical barrier (Figure 3A).

The remaining medicament was removed with 6\% Sodium Hypochlorite (Chlor-Xtra, Vista Dental, Racine, USA) with the Endovac negative pressure irrigation system (Kerr Corp., California, USA). Working length was reconfirmed (Figure $3 \mathrm{~B}$ ), the canal was dried with paper points and the open apex closed by packing a $4 \mathrm{~mm}$ increment of MTA (ProRoot MTA; DentsplySirona, Charlotte, USA). After the MTA set, the remainder of the root canal was obturated with

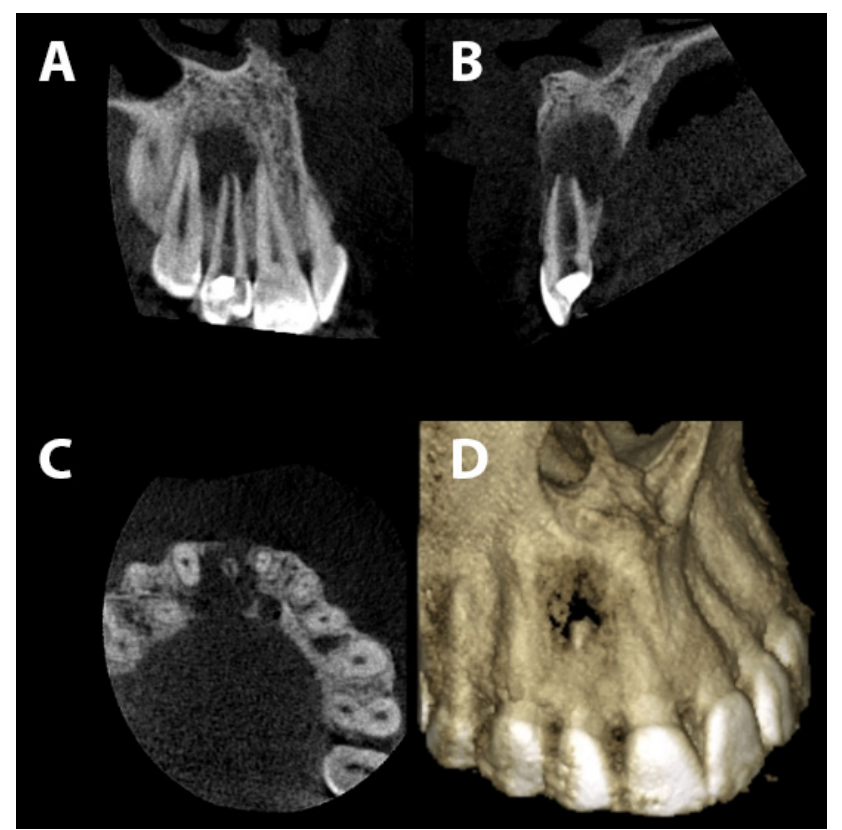

Figure 2. A limited field-of-view CBCT confirming the absence of a natural apical constriction, severe bone loss and perforation of buccal cortical plate: A) Coronal view, B) Sagittal view, C) Axial view and D) three-dimensional reconstruction. 


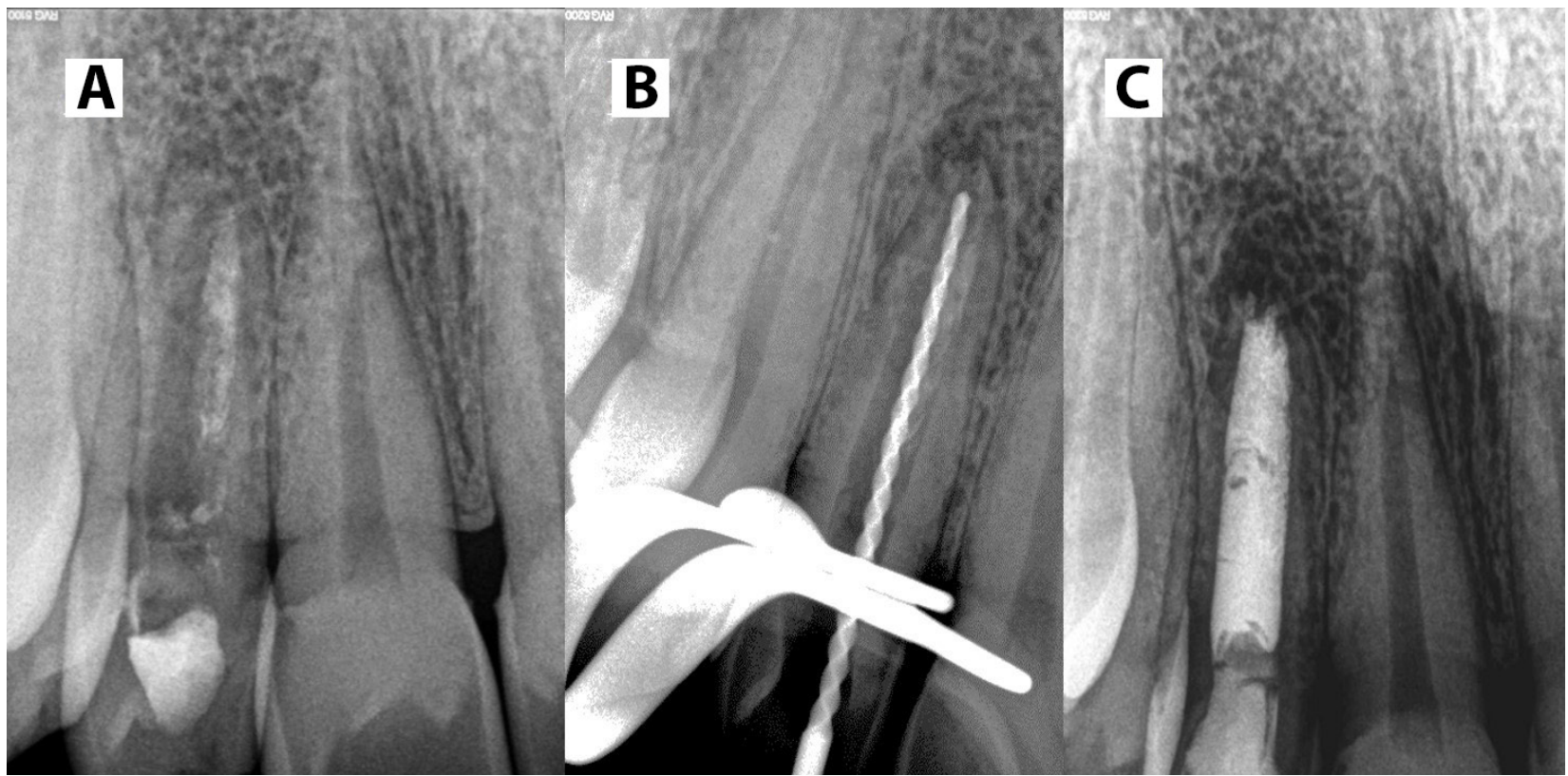

Figure 3. A) A two-year, follow-up periapical radiograph, revealing resolution of the periapical pathology B) working length confirmation with a large diameter $\mathrm{k}$-file C) immediate post-operative periapical radiograph.

heated gutta-percha delivered from a VDW Beefill backfill unit (VDW GmbH, Munich, Germany) (Figure 3C). The guttapercha was sealed at the orifice level with resin-modified glass ionomer (Vitrebond, 3M ESPE, St. Paul, USA) and the access cavity restored with Filtek Supreme composite (3M ESPE, Seefeld, Germany).

The patient was not committed to attend regular follow up visits, but was finally convinced to return for a follow-up evaluation. Four years after the initiation of the treatment, the patient was asymptomatic and the follow-up CBCT (Figure 4) showed bone fill around the right maxillary lateral incisor with complete healing of the buccal bone plate. The patient gave written consent for the use of the images and radiographs for publication.

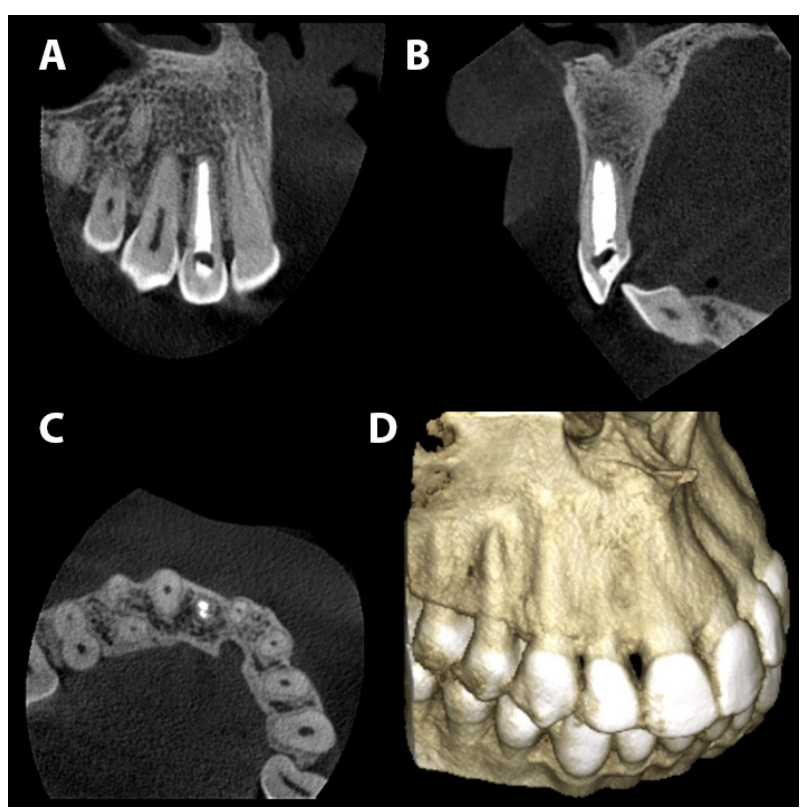

Figure 4. A 4-Year follow-up CBCT showing resolution of the apical area with bone fill: A) Coronal view, B) Sagittal view, C) Axial view and D) three dimensional reconstruction.

\section{DISCUSSION}

When pulpal necrosis occurs in immature permanent teeth with open apices, treatment options may include either root-end closure via apexification or regenerative endodontic procedures. ${ }^{1} \mathrm{Kim}$ et al. ${ }^{11}$ recommended that whilst either of the above-mentioned treatment approaches may be selected - teeth with near complete root formation (Stage 4 of Cvek's classification of root development, ${ }^{10}$ Figure 5) - may be better suited for apexification using MTA-barrier techniques. Whilst regenerative endodontics has the potential to produce vital periodontal-like tissues in the root canal space and encourage continued root formation, the literature still lacks high-level evidence supporting this approach ${ }^{12}$ with the majority of studies relying on case reports and case-series, and only a few randomized clinical trials with short-term follow-up. ${ }^{11}$ Additionally, fewer appointments are required to complete apexification procedures as compared to regenerative endodontic procedures. In the present case, the tooth was classified as Stage 4 according to Cvek's classification of root development and following discussion and informed consent with the patient, MTA apexification was considered the treatment of choice.

Appropriate cleaning, shaping, disinfection and filling of the root canal system is essential to the success of all nonsurgical endodontic treatment. ${ }^{13}$ Calcium hydroxide is commonly used as an intracanal medicament due to its bacteriostatic effects, due to the high $\mathrm{pH}$ value, and its ability to induce hard tissue formation. ${ }^{3}$ Triple antibiotic paste has been demonstrated to effectively eliminate bacteria when used in a protocol termed lesion sterilisation and tissue repair therapy (LSTR). ${ }^{7,14}$ When compared to $\mathrm{CH}$, TAP has been reported to demonstrate superior antimicrobial activity. ${ }^{15}$ For this reason, TAP may be selected as an alternative medicament in non-surgical endodontic cases where traditional $\mathrm{CH}$ paste fails to eliminate the symptoms. ${ }^{16,17}$ The healing demonstrated in the present case following the application of TAP supports these findings. 
(a)

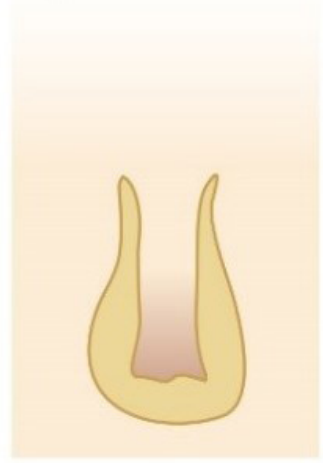

(b)

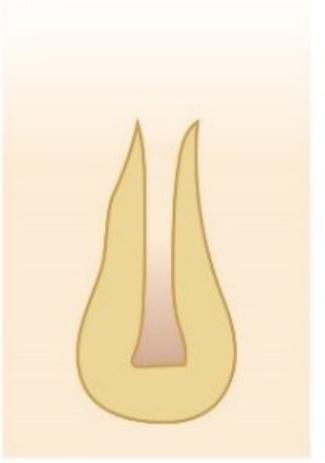

(c)

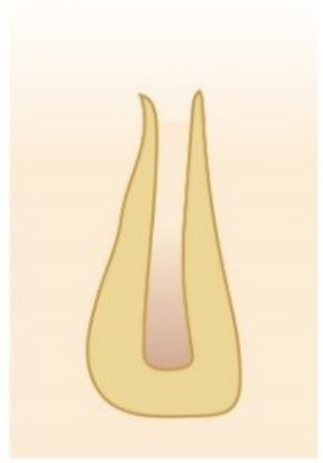

(d)

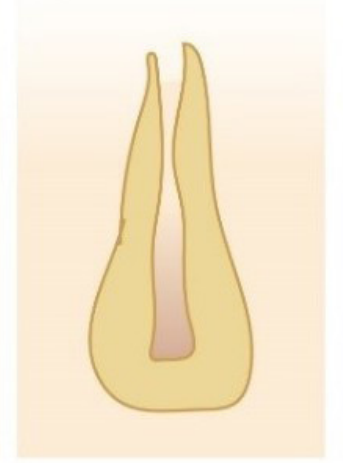

(e)

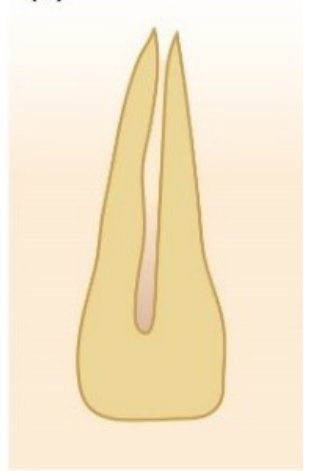

Figure 5. Cvek's classification of root development ${ }^{10}$

Crown discolouration may occur following apexification procedures due to either the minocycline present in $\mathrm{TAP}^{9}$ or the bismuth oxide found in MTA. ${ }^{4}$ In order to avoid this complication, some authors have suggested the use of Biodentine (Septodont, St. Maur-des-Fossés, France) instead of MTA, as this material does not contain bismuth oxide. ${ }^{18}$ Crown discolouration is however a known complication when selecting these materials and this risk should be well-communicated to the patient prior to treatment.

The diagnosis of large periapical lesions as radicular cysts on radiography alone remains a clinical challenge. ${ }^{19}$ Cone-beam computed tomography (CBCT) scanning has been proposed as a non-invasive method for differentiating between radicular cysts or periapical granulomas by measuring "grayscale value". ${ }^{20}$ Rosenberg et al. ${ }^{19}$ however reported that CBCT imaging is not a reliable method for diagnosing radicular cysts. The present case supports this assertion. The lesion associated with the right maxillary lateral incisor in the present case displayed many of the criteria used for the diagnosis of radicular cysts, which have been described as: welldefined uniform borders, a ballooning appearance, uniformly radiolucent lumen, perforation of the cortical plate and a size greater than $20 \mathrm{~mm}$ in diameter. ${ }^{19}$

Extensive bone loss surrounding open apices presents an additional clinical challenge to accurately seal the apex without extrusion of medicaments and other dental materials. A variety of matrices, such as collagen plugs, have been proposed as preliminary barriers to prevent over-extrusion of materials during apexification procedures. In recent years, platelet-rich fibrin (PRF) has been used as biocompatible, economical alternative barrier material that can additionally promote angiogenesis and wound healing. ${ }^{21}$ The use of barrier methods may however be technique sensitive. The healing of the periapical bone in the present case provided the clinician with an apical stop- therefore not necessitating the use of a preliminary apical barrier or the necessity of apical surgery with retrograde filling.

\section{CONCLUSION}

The present case demonstrates the successful nonsurgical endodontic treatment of a maxillary lateral incisor, classified as Cvek Stage 4 of root development, using TAP medicament and conventional MTA-barrier apexification.
Clinicians should be aware that alternative inter-appointment medicaments, such as TAP, may be beneficial in cases where conventional $\mathrm{CH}$ paste proves unsuccessful and that large periapical lesions of endodontic origin may heal sufficiently with non-surgical endodontic treatment alone and no surgical intervention.

\section{Acknowledgements}

None. The authors declare no conflict of interest exists.

\section{References}

1. Shabahang S. Treatment options: Apexogenesis and apexification. Pediatr Dent. 2013; 35: 125-8.

2. Sheely E, Roberts G. Use of calcium hydroxide for apical barrier formation and healing in non-vital immature permanent teeth: a review. Br Dent J. 1997; 183: 241-6.

3. Andreasen J, Farik B, Munksgaard E. Long-term calcium hydroxide as a root canal dressing may increase risk of root fracture. Dent Traumatol. 2002; 18: 134-7.

4. Parirokh M, Torabinejad M. Mineral Trioxide Aggregate: A Comprehensive Literature Review-Part III: Clinical Applications, Drawbacks, and Mechanism of Action. J Endod. 2010; 36: 400-13.

5. Modena K, Casas-Apayco L, Atta M, Costa C, Hebling J, Sipert C, et al. Cytotoxicity and biocompatibility of direct and indirect pulp capping materials. J Appl Oral Sci. 2009; 17: 544-54.

6. Bajwa N, Jingarwar M, Pathak A. Single Visit Apexification Procedure of a Traumatically Injured Tooth with a Novel Bioinductive Material (Biodentine). Int $\mathrm{J}$ Clin Pediatr Dent. 2015; 8: 58-61.

7. Sato I, Kota K, Iwaku M, Hoshino E. Sterilization of infected root-canal dentine by topical application of a mixture of ciprofloxacin , metronidazole and minocycline in situ. Int Endod J. 1996; 29: 118-24.

8. Trope M. Regenerative potential of dental pulp. Pediatr Dent. 2008; 30: 206-10.

9. Huang G. A paradigm shift in endodontic management of immature teeth: Conservation of stem cells for regeneration. J Dent. 2008; 36: 379-86.

10. Cvek M. Prognosis of luxated non-vital maxillary incisors treated with calcium hydroxide and filled with gutapercha. Dent Traumatol. 1992; 8: 45-55.

11. Kim S, Malek M, Sigurdsson A, Lin L, Kahler B. Regenerative endodontics: a comprehensive review. Int 
Endod J. 2018; 51: 1367-88.

12. Kontakiotis E, Filippatos C, Agrafioti A. Levels of evidence for the outcome of regenerative endodontic therapy. J Endod. 2014; 40: 1045-53.

13. Soares J, Santos S, Silveira F, Nunes E. Nonsurgical treatment of extensive cyst-like periapical lesion of endodontic origin. Int Endod J. 2006; 39: 566-75.

14. Hoshino E, Kurihara-Ando N, Sato I, Uematsu H, Sato $\mathrm{M}$, Kota K, et al. In-vitro antibacterial susceptibility of bacteria taken from infected root dentine to a mixture of ciprofloxacin, metronidazole and minocycline. Int Endod J. 1996; 29: 125-30.

15. Madhubala M, Srinivasan N, Ahamed S. Comparative Evaluation of Propolis and Triantibiotic Mixture as an Intracanal Medicament against Enterococcus faecalis. J Endod. 2011; 37: 1287-9.

16. Er K, Kuştarci A, Özan Ü, Taşdemir T. Nonsurgical Endodontic Treatment of Dens Invaginatus in a Mandibular Premolar with Large Periradicular Lesion: A Case Report. J Endod. 2007; 33: 322-4.

17. Kusgoz A, Yildirim T, Er K, Arslan I. Retreatment of a Resected Tooth Associated with a Large Periradicular Lesion by Using a Triple Antibiotic Paste and Mineral Trioxide Aggregate: A Case Report with a Thirty-month
Follow-up. J Endod. 2009; 35: 1603-6.

18. Vidal K, Martin G, Lozano O, Salas M, Trigueros J, Aguilar G. Apical Closure in Apexification: A Review and Case Report of Apexification Treatment of an Immature Permanent Tooth with Biodentine. J Endod. 2016; 42: 730-4.

19. Rosenberg P, Frisbie J, Lee J, Lee K, Frommer H, Kottal S, et al. Evaluation of Pathologists (Histopathology) and Radiologists (Cone Beam Computed Tomography) Differentiating Radicular Cysts from Granulomas. J Endod. 2010; 36: 423-8.

20. Simon J, Enciso R, Malfaz J, Roges R, Bailey-Perry M, Patel A. Differential Diagnosis of Large Periapical Lesions Using Cone-Beam Computed Tomography Measurements and Biopsy. J Endod. 2006; 32: 833-7.

21. Sharma S, Sharma V, Passi D, Srivastava D, Grover S, Dutta S. Large Periapical or Cystic Lesions in Association with Roots Having Open Apices Managed Nonsurgically Using 1-step Apexification Based on Platelet-rich Fibrin Matrix and Biodentine Apical Barrier: A Case Series. J Endod. 2018; 44: 179-85.

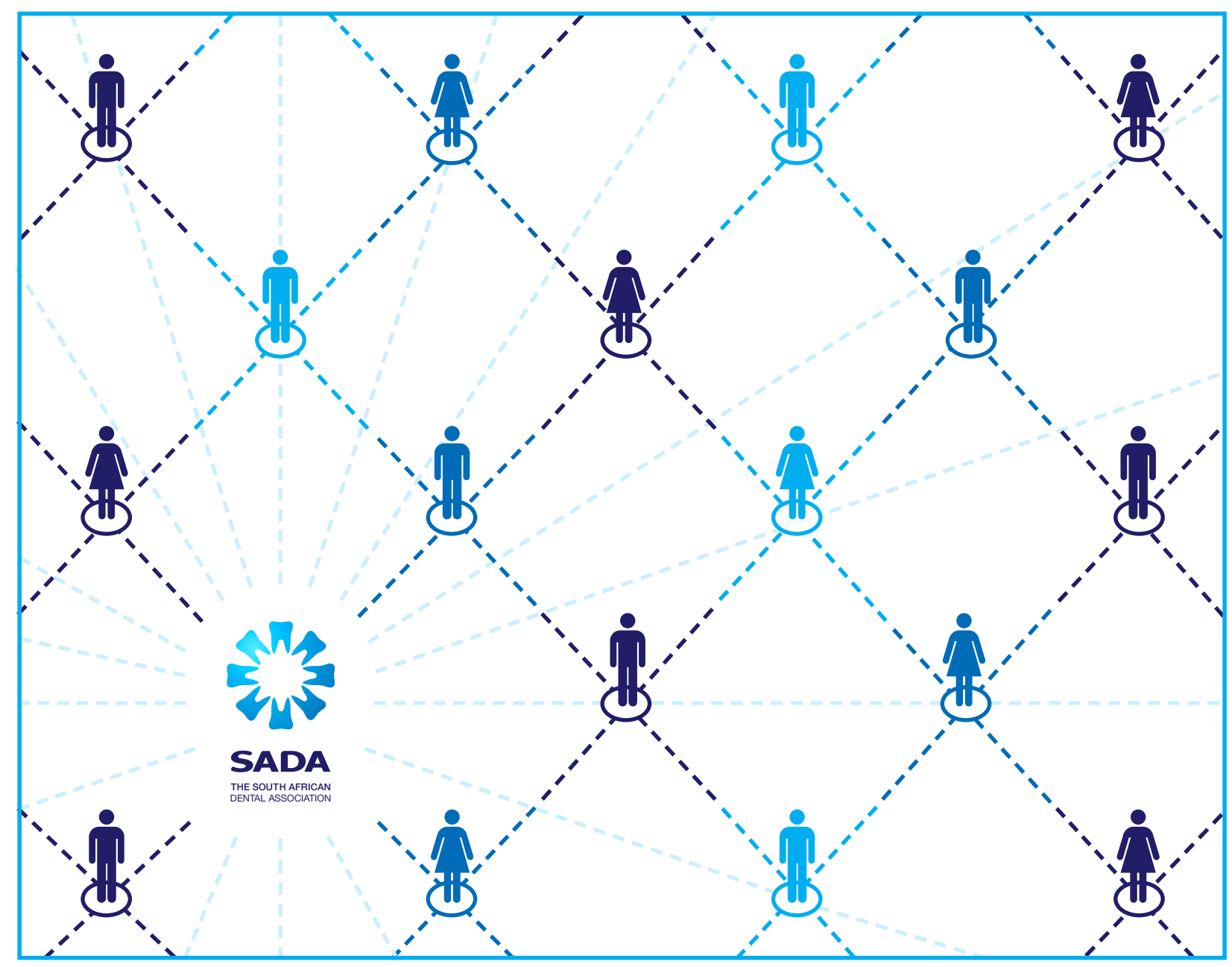

